\title{
Dentes supranumerários e suas consequências na dentadura mista: relato de caso clínico
}

Supernumerary teeth and their consequences in mixed dentition: clinical case report

Dientes supernumerarios y sus consecuencias en la dentición mixta: reporte de un caso clínico

Luciana Rodrigues Chaves ORCID: https://orcid.org/0000-0001-7857-8193 Centro Universitário Fametro, Brasil E-mail: lucianarodrigueschaves30@gmail.com Maria Gerlane Germano de Carvalho ORCID: https://orcid.org/0000-0002-0635-2096 Centro Universitário Fametro, Brasil E-mail: gegermano03@gmail.com Michele di Benedetto

ORCID: https://orcid.org/0000-0003-1532-0245 Centro Universitário Fametro, Brasil E-mail: benedettom61@gmail.com

Karina Alessandra Guimarães Barbosa ORCID: https://orcid.org/0000-0003-4933-6421 Centro Universitário Fametro, Brasil E-mail: karina.barbosa@fametro.edu.br

Gabriel Catunda de Souza ORCID: https://orcid.org/0000-0002-0806-9841 Centro Universitário Fametro, Brasil

E-mail: gabriel.catunda@fametro.edu.br

Joyce de Figueiredo Meira ORCID: https://orcid.org/0000-0001-5156-236X Faculdade FAIPE, Brasil E-mail: joycefmeira@fametro.edu.br

Nayhane Cristine da Silva de Oliveira ORCID: https://orcid.org/0000-0003-2056-5853 Centro Universitário Fametro, Brasil

E-mail: nayhane.oliveira@fametro.edu.br

Gabriela de Figueiredo Meira

ORCID: https://orcid.org/0000-0002-8285-8769 Universidades Federal de Santa Maria, Brasil E-mail: gabrielameira1@ hotmail.com

\begin{abstract}
Resumo
Objetivo: O caso clínico descreve a presença de um dente supranumerário localizado entre os incisivos superiores em paciente pediátrico, sexo masculino, com 9 anos e 5 meses de idade. Metodologia: Partindo da permissão da paciente por meio do Termo de Consentimento Livre e Esclarecido (TCLE) em partilhar sua imagem para devido fim, o presente estudo trata de um relato de caso clínico de cunho analítico descritivo (Pereira et al., 2018). Relato de Caso Clínico: Paciente do sexo masculino, J.V.S.D., de 9 anos e 5 meses de idade, compareceu à clínica odontológica infantil do Centro Universitário Fametro (CEUNI) para avaliação clínica durante a anamnese, o responsável relatou que a queixa principal era que a criança apresentava um dente a mais na boca. Negou sensação dolorosa na região, assim como, sangramentos. Considerações Finais: O caso clínico mostrou a importância da anamnese, exame clínico e de imagem para fechar um bom diagnóstico e plano de tratamento, culminando no sucesso clínico.
\end{abstract}

Palavras-chave: Dentes; Supranumerários; Pediatria; Desenvolvimento.

\begin{abstract}
Objective: The clinical case describes the presence of a supernumerary tooth located between the upper incisors in a pediatric male patient, aged 9 years and 5 months. Methodology: Based on the patient's permission through the Informed Consent Form (FICF) to share her image for the proper purpose, this study is a clinical case report of a descriptive analytical nature (Pereira et al., 2018). Clinical Case Report: Male patient, JVSD, 9 years and 5 months old, attended the children's dental clinic at Centro Universitário Fametro (CEUNI) for clinical evaluation during the anamnesis, the responsible person reported that the main complaint was that the child had one more tooth in his mouth. Denied painful sensation in the region, as well as bleeding. Final Considerations: The clinical case showed the
\end{abstract}


importance of anamnesis, clinical and imaging examination to conclude a good diagnosis and treatment plan, culminating in clinical success.

Keywords: Supernumerary; Teeth; Pediatrics; Development.

\section{Resumen}

Objetivo: El caso clínico describe la presencia de un diente supernumerario ubicado entre los incisivos superiores en un paciente pediátrico masculino, de 9 años y 5 meses. Metodología: Con base en el permiso de la paciente a través del Término de Consentimiento Informado (FICF) para compartir su imagen con el propósito adecuado, este estudio es un reporte de caso clínico de naturaleza analítica descriptiva (Pereira et al., 2018). Reporte de Caso Clínico: Paciente masculino, JVSD, 9 años y 5 meses de edad, acudió a la clínica dental infantil del Centro Universitário Fametro (CEUNI) para evaluación clínica durante la anamnesis, el responsable informó que la principal queja era que el niño tenía una más diente en la boca. Se niega la sensación de dolor en la región, así como sangrado. Consideraciones finales: El caso clínico mostró la importancia de la anamnesis, el examen clínico y de imagen para concluir un buen diagnóstico y plan de tratamiento, culminando en el éxito clínico.

Palabras clave: Dientes; Supernumerarios; Pediatría; Desarrollo.

\section{Introdução}

Os dentes supranumerários são manifestações de anomalia congênita de desenvolvimento do número de dente (Nunes et al., 2015). Estes são encontrados na literatura geralmente como um germe dental que se desenvolvem próximo a uma lâmina de um dente adjacente permanente. Nesses casos, o Cirurgião-Dentista poderá solicitar exames complementares para melhor diagnosticar um tratamento. Frequentemente, o Raio-X Panorâmico pode dar uma excelente visualização de toda a arcada dental. Tais dentes podem aparecer na dentição decídua ou mista, sendo mais comum na permanente (Santos et al., 2020).

Sabemos que o mais comum são os mesiodens, que se desenvolvem na região de incisivos superiores e sua ocorrência pode ser de forma unitária ou múltipla, na mandíbula, na maxila (Neville et al., 2009).

De acordo com Loreto et al., (2015), ao ouvir a queixa principal o cirurgião-dentista (CD) poderá diagnosticar com base nos relatos e no exame clínico as possíveis causas da visita do paciente. No caso de dentes supranumerários que não estejam visíveis, é necessária a solicitação de exames de imagens, como raio-x panorâmico ou, em alguns casos a tomografia computadorizada. Não se deve subestimar o efeito tranquilizador que causam a boa relação profissional-paciente e o diálogo pré-operatório, no qual se procura transmitir segurança e confiança (MedeiroS, 2003)

Para os ortodontistas, os exames de imagem fazem parte do seu kit inicial de tratamento, podendo ser repetido em qualquer época durante a evolução do tratamento para melhor acompanhar o desenvolvimento (Martorelli et al., 2018). Nasseh \& Al Rawi, (2018) mostraram em seu trabalho as várias aplicações da tomografia computadorizada na área odontológica, entre elas, localização das características anatômicas e anormalidades de desenvolvimento, sem ampliação e sobreposições e contraste bastante satisfatório, auxiliando o correto diagnóstico e planejamento do tratamento.

A radiografia panorâmica também oferece uma imagem bidimensional, é indicada para o planejamento de procedimentos em relação aos dentes impactados pois oferece uma visão geral dos arcos, possibilitando a correlação do dente impactado com os demais dentes e estruturas próximas, mas não permite a localização precisa dos dentes (Boeddinghaus \& Whyte, 2008; Meara, 2012).

Como opção de tratamento, a remoção cirúrgica é uma das indicações em casos de dentes supranumerários, devido a uma série de transtornos que esses dentes dependendo da localização poderão causar. Outra forma mais conservadora de tratar seria o acompanhamento rotineiro através dos exames solicitados pelo cirurgião-dentista (Martorelli et al., 2018).

Quando a indicação é sugerida pelo Cirurgião dentista e este paciente optar em não realizar, profissional deverá se munir de documentos comprobatórios, relatando que durante o tratamento ou consulta de rotina informou ao paciente que seria indicado a remoção cirúrgica e o mesmo optou em não realizar (Loreto et al., 2015). 
Existem consequências relacionadas a presença de múltiplos dentes supranumerários, as principais são erupção retardada, impacção dental, deslocamento dos dentes permanentes durante a erupção, reabsorção radicular e formação cística. (Açıkgöz et al., 2006; Khalaf et al., 2018; McCoy, 2012)

De acordo com Moura et al., (2013), o paciente precisa estar ciente dos possíveis problemas futuros que um supranumerário poderá causar como: Rotação, apinhamento dental, bem como outros problemas ortodônticos e oclusais, cárie e severos danos à saúde gengival, risco de patologias como abcessos e cistos odontogênicos, além do comprometimento estético.

Dessa forma, o objetivo desse artigo é relatar um caso clínico de paciente pediátrico com dente supranumerário na maxila, bem como sua conduta de tratamento e aspectos relacionados ao elemento, como sua incidência, plano de tratamento e complicações associadas.

\section{Metodologia}

Partindo da permissão da paciente por meio do Termo de Consentimento Livre e Esclarecido (TCLE) em partilhar sua imagem para devido fim, o presente estudo trata de um relato de caso clínico de cunho analítico descritivo (Pereira et al., 2018), realizado na clínica odontológica do CEUNI Fametro, na disciplina de estágio supervisionado em Clínica Integrada II, do curso de graduação do Centro Universitário Fametro.

\section{Resultados e Discussão}

Paciente do sexo masculino, J.V.S.D., de 9 anos e 5 meses de idade, compareceu à clínica odontológica infantil do Centro Universitário Fametro (CEUNI) para avaliação clínica durante a anamnese, o responsável relatou que a queixa principal era que a criança apresentava um dente a mais na boca. Negou sensação dolorosa na região, assim como, sangramentos (Figura 1)

Figura 1: A) Fotografia intrabucal - aspecto frontal; B) Fotografia intrabucal da arcada superior - vista palatina.

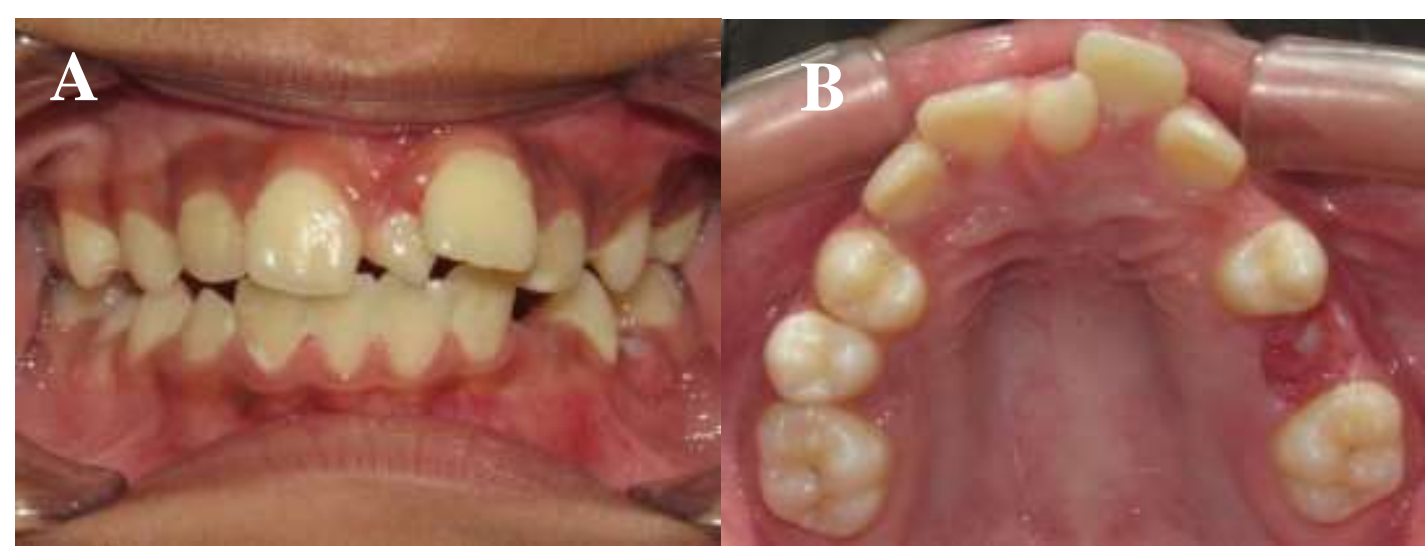

Fonte: Autores.

Em relação das doenças sistêmicas, o responsável afirmou não haver qualquer alteração que pudesse interferir ou contraindicar o procedimento cirúrgico. Anomalias dentárias ou casos semelhantes foram negados entre outros familiares. Declarou que a higiene oral era realizada através de escovação duas vezes ao dia com dentifrício fluoretado e, às vezes, realizava o uso de fio-dental e exanguante bucal.

Ao exame físico intra-oral, foi observado ausência dos dentes 13, 23 e 25, sem necessidade de qualquer tratamento dentário prévio e gengiva com aspecto saudável. Dos elementos presentes, todos eram permanentes e com formação radicular 
completa. Entre os incisivos centrais superiores, havia a presença de um elemento dentário extra, sugestivo de dente supranumerário do tipo Mesiodente (mesiodens). A radiografia periapical foi realizada e a radiografia panorâmica foi então solicitada.

Ao retornar, o exame radiográfico foi apresentado e analisado (Figura 2). As estruturas ósseas apresentavam-se sem alterações e os elementos $18,17,13,23,25,27,28,38,37,33,45,47,48$ em processo de formação/erupção. Foi confirmada ainda a presença do mesiodente.

Figura 2: A) Radiografia panorâmica; B) Radiografia periapical da região dos incisivos superiores.

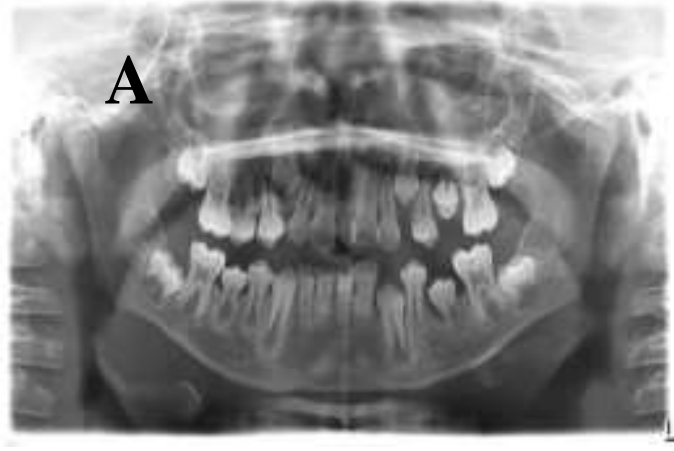

Fonte: CIMO.

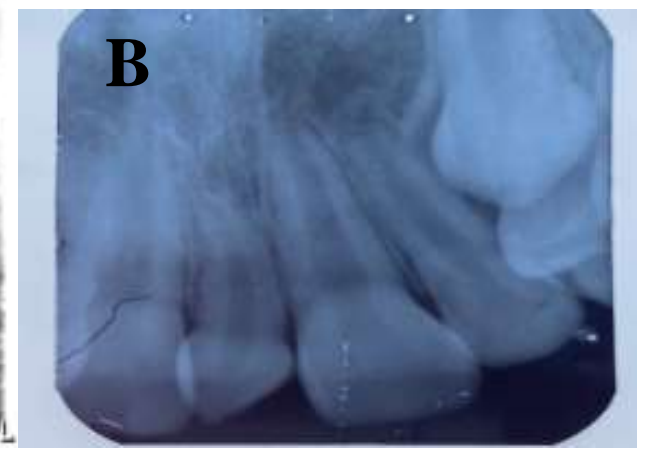

Fonte: Clínica odontológica CEUNI-Fametro.

O tratamento proposto para o caso foi o de remoção cirúrgica do dente supranumerário com a finalidade de permitir o alinhamento dentário na arcada para posterior tratamento ortodôntico, além de melhorar o resultado estético e permitir um retorno social, aumentando ainda a auto-estima da criança. Para o procedimento cirúrgico, inicialmente, foi realizada a antissepsia extra-oral com uso de clorexidina 2\%. Logo após a técnica anestésica foi executada, com aplicação de anestésico tópico benzotop benzocaina200mg/g(nova DFL®). O anestésico de escolha foi lidocaína $2 \%$ com vasoconstritor epinefrina 1:100.000 (nova DFL®).

Em seguida, iniciou-se a sindesmotomia com descolador de molt 2/4, posteriormente a luxação e remoção do elemento foram realizadas com alavancas Seldin e fórceps $n^{\circ} 01$ com movimentos de intrusão, rotação e tração com as devidas precauções para não luxar os elementos adjacentes. Após a remoção, irrigou-se o soro fisiológico a 0,9\%. Ao final, o responsável foi instruído a administrar analgésico por 24 horas, em caso de dor ou incômodo (Figuras 3).

Figura 3: A) Aspecto cirúrgico após a exodontia do elemento supranumerário; B) Mesiodente removido.

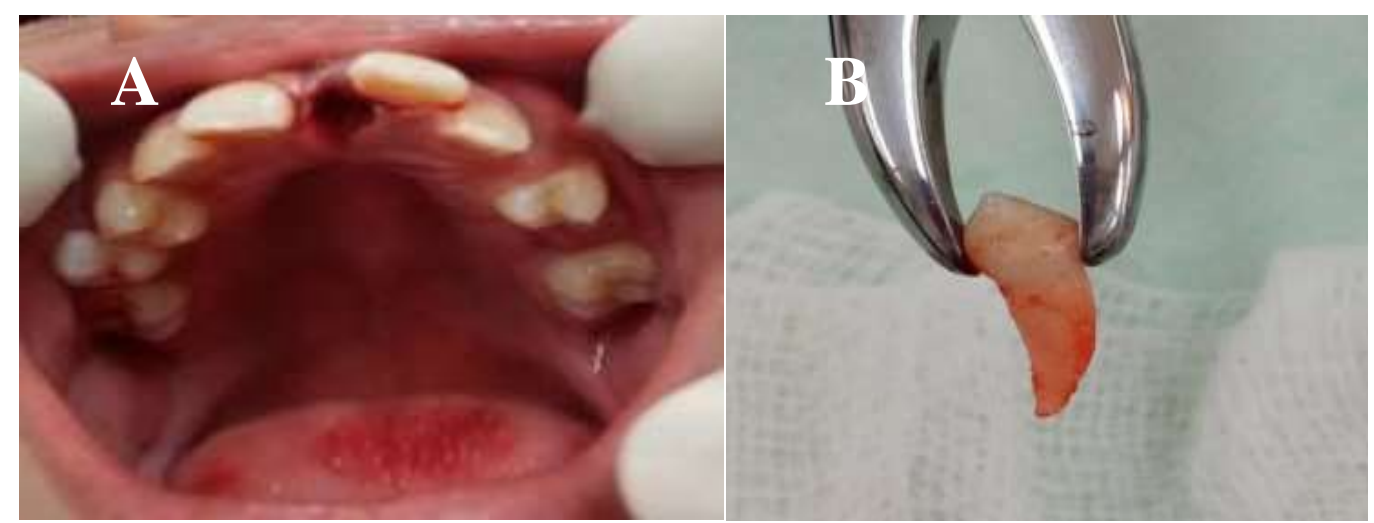

Fonte: Autores. 
Das recomendações pós-operatórias, foi passado ao responsável os cuidados quanto a higienização, manipulação da cavidade bucal da criança e sua alimentação. Decorrido o período pós-operatório, e foi analisado um bom aspecto cicatricial na região da exodontia, além de nenhuma alteração na dentição adjacente (Figura 4). O paciente está em acompanhamento e será iniciada a terapia ortopédica.

Figura 4: Resultado final do caso.

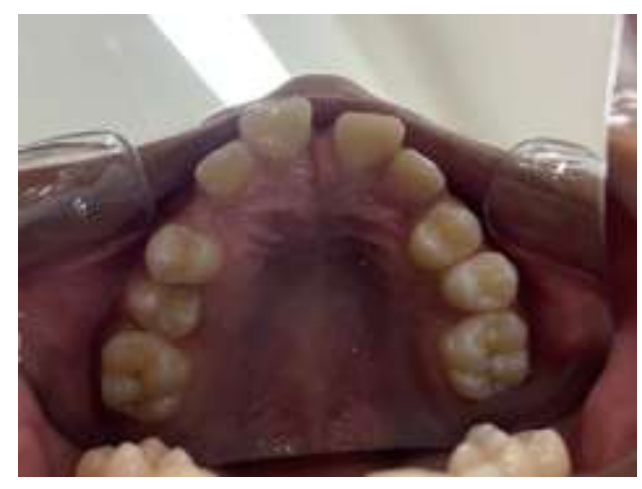

Fonte: Autores.

\section{Discussão}

A ocorrência de dentes supranumerários pode ser vista em qualquer região da cavidade oral, seja em maxila ou mandíbula, podendo ser de um ou vários dentes. Dias et al., (2019) mostraram que o mesiodente é o tipo mais comum de dente supranumerário, podendo ser único, múltiplo, bilateral ou unilateral, prevalente em cerca de $0,15 \%$ e $1,9 \%$ da população e que ocorre mais em homens do que em mulheres. Na dentição decídua, os autores mostraram que o diagnóstico precoce da condição leva à prevenção de complicações ao sistema estomatognático, sendo o uso de exames complementares como: radiográficas periapicais, panorâmicas e oclusais, além da tomografia computadorizada de extrema importância no diagnóstico. No presente caso o paciente possuía um mesiodente na dentição mista que levava a criança a ter vergonha de sorrir. Sendo o tratamento de escolha a remoção cirúrgica.

Em conformidade, Nunes et al., (2015) demonstraram que a etiologia dos supranumerários é multifatorial, podendo se dá através de fatores ambientais, genéticos ou na fase de desenvolvimento dentário. Além disso, estes elementos recebem classificação conforme sua morfologia, sendo: suplementares - quando possuem características de dentes normais e rudimentares, ao adquirirem formas diferentes como dentes conóides (formato de cone), trabeculados (formato de barril) e, até mesmo com formato de odontomas. Podem estar localizados entre os incisivos centrais superiores, onde são denominados "mesiodens", entre molares (paramolares), entre pré-molares (parapremolares) e distomolares aos que se manifestarem após o terceiro molar.

Durante a anamnese não foi relatado nenhum outro caso na família de anomalias dentárias.

Moro et al., (2020) estudaram a prevalência de dentes supranumerários nos pacientes atendidos na disciplina de Cirurgia e Traumatologia Bucomaxilofacial de uma Clínica-Escola Odontológica no estado do Rio Grande do Sul. Dos casos encontrados, $61,54 \%$ foram vistos em maxila, o que concorda com a maioria das literaturas, houve ainda uma maior predileção pelo sexo feminino, apesar de muitos apontarem o sexo masculino como o mais acometido. A região de pré-molar foi a que mais apresentou os supranumerários. Ademais, do número de dentes por indivíduo, $60 \%$ apresentava apenas um elemento a mais. Da amostra, foi observado ainda que uma pequena parte apresentava radiografia panorâmica, de extrema importância para o diagnóstico, sugerindo que fatores socioeconômicos podem ter interferido nesse quesito. 
Um outro estudo foi realizado por Negrete et al., (2015) na Universidade Cidade de São Paulo. Foram analisadas 1117 pacientes através de radiografias panorâmicas do acervo de ortodontia da faculdade. Desse total, em 18 (1,61\%) houve a presença de dentes supranumerários. $\mathrm{O}$ índice por gênero foi de 1,9\% para o sexo feminino e 1,2\% para o masculino, sem muita significância. Mais da metade dos casos foi encontrado em maxila $(66,7 \%)$ corroborando com grande parte da literatura quanto à localização. Da região bucal, a região foi a mais apontada com o tipo mesiodente.

Khalaf et al., (2018) realizaram uma revisão de literatura de dentes supranumerários na região de pré-molar. Os autores mostraram que a região possui características únicas quando comparado às outras, além da etiologia, consequência clínica, diagnóstico e tratamento. A revisão indicou ainda que ao contrário dos demais, a prevalência é em mandíbula e do tipo suplementar sendo a maioria diagnosticada em exames radiográficos devido ao seu caráter assintomático. Das consequências, se destacam a formação de cistos, transposição e má oclusão.

Teslenco et al., (2017) trouxe um relato de caso de dentes supranumerários em mandíbula, bilateralmente, em sexo feminino, diferente da prevalência comum em maxila. A cirurgia ocorreu na Universidade Federal do Mato Grosso do Sul em duas etapas, foram realizadas tomadas radiográficas e o planejamento que consistiu em remover os elementos em duas datas distintas. Ao final, o procedimento foi realizado sem intercorrências, não sendo necessário o uso de antibioticoterapia, prevenindo ainda problemas futuros como: reabsorções, patologias e necrose de elementos adjacentes. Esses dados vão de acordo com estudo de Moura et al., (2021), onde a conduta geral é a remoção dos elementos supranumerários com a finalidade de deixar o sorriso mais harmônico e causar menor transtorno à infância da criança, já que um dente a mais pode ser uma fator de baixa autoestima e aceitação social. No relato de caso, os autores trouxeram uma situação semelhante ao caso apresentado, com paciente do sexo masculino também acometido por dente supranumerário entre os incisivos centrais causando um diastema na região, tratando-se de um mesiodente.

Já Araújo et al., (2021) atribuiu que após o diagnóstico de supranumerário, a intervenção cirúrgica é a mais indicada a fim de evitar complicações futuras, além do fator ortodôntico, visto que um dente supranumerário pode interferir no desenvolvimento de uma oclusão correta. Outros fatores incluem a estética, fonética e os fatores sociais. Todavia, em casos em que o diagnóstico é favorável e apresenta baixas taxas de complicações. É recomendado um acompanhamento a uma maior idade do paciente para realização do procedimento, até que este possa estar preparado para uma cirurgia mais invasiva, evitando também possíveis danos a raízes de outros elementos dentários. No presente caso, a remoção cirúrgica foi adotada, visto que, o paciente já se encontrava com a dentição permanente e formação radicular quase completa.

Cruz et al., (2015) mostrou uma conduta clínica perante dentes supranumerários localizados em pré-maxila na qual os autores mostraram que a conduta clínica consiste na remoção cirúrgica ou reposicionamento ortodôntico dos elementos a mais existentes. Alguns casos são contraindicados à remoção, mas devem ser analisados pelo cirurgião-dentista. No caso clínico apresentado, o mesiodente comprometia a estética do paciente, por isso a melhor indicação foi a remoção.

\section{Considerações Finais}

O caso clínico mostrou a importância da anamnese, exame clínico e de imagem para fechar um bom diagnóstico e plano de tratamento, culminando no sucesso clínico. É importante que o cirurgião-dentista esteja capacitado e preparado para avaliar e receber casos de anomalias dentárias, principalmente as de número, como é o caso do dente supranumerário e entender a influência da anomalia na qualidade de vida, estética e impacto social no indivíduo, principalmente na infância, onde traumas podem percorrer por toda a vida. 


\section{Referências}

Açıkgöz, A., Açıkgöz, G., Tunga, U., \& Otan, F. (2006). Characteristics and prevalence of non-syndrome multiple supernumerary teeth: A retrospective study.Dentomaxillofacial Radiology, 35(3), 185-190. https://doi.org/10.1259/dmfr/21956432.

Araújo, B. M., et al. (2021). Conduta clínica para tratamento de dentes supranumerários: Relato de caso clínico. Research, Society and Development, 10(6), e31110615807-e31110615807.

Boeddinghaus, R., \& Whyte, A. (2008). Current concepts in maxillofacial imaging. European Journal of Radiology, 66(3), 396-418.

Cruz, M. C. C., et al. (2017). Diagnóstico e conduta clínica perante dentes supranumerários localizados em pré-maxila: relato de um caso. Revista de Odontologia da Universidade, Cidade de São Paulo, 27(3), 258-262.

Dias, G. F., et al. (2019). Diagnóstico e tratamento de dentes supranumerários na clínica infantil-relato de caso. Revista CEFAC, 21. https://doi.org/10.1016/j.ejrad.2007.11.019.

Khalaf, K., Al Shehadat, S., \& Murray, C. A. (2018). A Review of Supernumerary Teeth in the Premolar Region. International Journal of Dentistry, 2018, 1-6. https://doi.org/10.1155/2018/6289047.

Loreto, A. F. V., Kelmer, F., \& De Oliveira, R. V. (2015). Supranumerário na região de mandíbula interferindo na oclusão: diagnóstico, planejamento e tratamento de um caso clínico. Revista uningá review, 21(1).

Martorelli, S. B. F., Lacerda, E. P. M., \& Andrade, F. B. M. (2018). Microtooth supernumerary included in Zygomatic root. RGO-Revista Gaúcha de Odontologia, 66, 82-87.

McCoy, J. M. (2012). Complications of Retention: Pathology Associated with Retained Third Molars. Atlas of the Oral and Maxillofacial Surgery Clinics, 20(2), 177-195. https://doi.org/10.1016/j.cxom.2012.06.002.

Meara, D. J. (2012). Evaluation of Third Molars: Clinical Examination and Imaging Techniques. Atlas of the Oral and Maxillofacial Surgery Clinics, 20(2), 163-168. https://doi.org/10.1016/j.cxom.2012.07.001.

Medeiros, P. J. (2003). Cirurgia dos dentes inclusos: extração e aproveitamento. Liv.Santos, 147.

Moro, F. A. P., Boneti, M. N., \& Costa, A. A. I. (2020). Prevalência de dentes supranumerários em pacientes atendidos em uma clínica-escola de odontologia do norte do Rio Grande do Sul. Revista Perspectiva, 44(165), 171-178.

Moura, A. B. R., et al. (2021). Identificação e remoção cirúrgica de dentes supranumerários: relato de caso. Archives Of Health Investigation, 10(5), 725-728.

Nasseh, I., \& Al-Rawi, W. (2018). Cone Beam Computed Tomography. Dental Clinics of North America, 62(3), 361-391. https://doi.org/10.1016/j.cden.2018.03.002.

Negrete, D., et al. (2017). Prevalência de dentes supranumerários em pacientes de Ortodontia. Revista de Odontologia da Universidade Cidade de São Paulo, 27(1), 6-13.

Neville et al. (2009). Patologia oral e maxilofacial. (3a ed.), Elsevier, 972.

Nunes, K. M., et al. (2017). Dente supranumerário: revisão bibliográfica e relato de caso clínico. Revista de Odontologia da Universidade Cidade de São Paulo, 27(1), 72-81.

Pereira, A. S., Shitsuka, D. M., Parreira, F. J., \& Shitsuka, R. (2018). Metodologia da pesquisa científica. UFSM.

Santos, V. C. D. de O., Castro, B. A. M. de, Martins, V. da M., Paranhos, L. R., Silva, G. R. da, Dietrich, L., \& Costa, M. D. M. de A. (2021). Múltiplos dentes impactados em paciente não sindrômico. Research, Society and Development, 10(1), e49110111626. https://doi.org/10.33448/rsd-v10i1.11626.

Santos, W. B., et al. (2020). Remoção cirúrgica de três supranumerários inclusos em mandíbula: Relato de caso. Revista Eletrônica Acervo Saúde, 40, e2397e2397.

Teslenco, V B., Gaetti-Jardim, E. C., \& Da Silva, J. C. L. (2017). Supranumerários bilaterais em mandíbula: relato de caso. Archives Of Health Investigation, $6(3)$. 